\title{
Working while sleep-deprived: not just a problem for residents
}

$\mathrm{C}$ ompletion of medical training does not render doctors immune to problems related to sleep deprivation. Evidence suggests that prolonged work periods without sleep can increase the risk of complications for patients and may be detrimental to physicians' health. ${ }^{1,2}$ For residents and medical students, such evidence has led to strict limits on maximum work hours, ${ }^{2,3}$ but for practising physicians the problem is largely ignored.

A 10-year study noted that the rates of surgical complications were substantially elevated among patients whose surgeon had had less than six hours of uninterrupted sleep the night before. ${ }^{4}$ Indeed, doctors in practice, being older, may be more susceptible than trainees to the effects of sleep deprivation. Moreover, unlike trainees, whose practice is supervised, doctors in practice often have no one overlooking them to catch their mistakes. A 2010 study noted that at least two nights' adequate sleep are needed following a working night on call for doctors to feel fully refreshed.

The problem may only be getting worse. Medical care today is more complex than in decades past. Patients with life-threatening conditions who decades ago would have died now survive because of medical innovations, new drugs and advanced technologies. Increasing complexity of care at the bedside or in the operating theatre places unprecedented cognitive and physical demands on doctors who oversee and deliver care in these environments. Sleep deprivation (i.e., less than six hours of uninterrupted sleep) from overnight call has been shown to cause a similar degree of impairment in judgment and motor performance as a blood alcohol level above $0.05 \%{ }^{6}$

Prolonged work hours are not limited to doctors involved in high-technology advanced care. A primary care physician who is up all night assisting at a birth or dealing with a patient in crisis may, because of sleep deprivation, be at increased risk for errors in judgment when seeing patients in the office the next day.

Unquestionably, there are substantial barriers to limiting work hours for all physicians. A 2009 US study examined the implications of applying the aviation industry's strategy for restriction of work hours for fatigue management to the medical system. The cost was estimated to be more than $\$ 1000000$ per patient life-year saved and would require a $71 \%$ increase in the US physician workforce and a $174 \%$ increase in the number of residents. ${ }^{7}$ This strategy was deemed neither feasible nor fiscally prudent. Moreover, increasing physician supply alone would not solve the problem of physician distribution and might force underserviced communities to choose between sleep-deprived care and no care.

Compliance with limits on work hours might prove another important barrier, as has been seen in the aviation and trucking industries. We doctors ourselves are part of this problem. We need to shift our professional culture. Long periods on call should not be accepted as routine or a source of pride. Instead, we must admit that working while impaired from sleep deprivation is neither normal nor acceptable. Those of us who remain overconfident that we can continue to perform our duties properly with inadequate sleep should imagine the reaction if we were made to seek informed consent from each of our patients to accept treatment under these conditions.

Solving this complex problem begins with acknowledging the issue and initiating a dialogue. Next, existing efforts to address the problem must be evaluated. Some hospitals, departments and group practices have implemented innovative approaches to work scheduling. Strategies may include strict policies for going home after call, refraining from booking procedures or clinics the day following call, reorganizing call schedules to allow for more physician coverage or moving to shift work. These approaches may be more feasible in some settings and among some specialties than others. Creative alternatives will be needed and should be evaluated for effects on acceptability, continuity and quality of care.

Ultimately, licensing, accreditation, insurance and government institutions need to establish minimum best practice standards for maximum work and minimum uninterrupted sleep hours. Authorities need to examine current incentives and disincentives for extended hours of work to ensure that the system does not perversely make it harder for physicians to work more restricted hours. Patients, especially in acute care settings, need assurance of receiving quality care from doctors who are not sleep deprived, whatever their status or experience.

Noni E. MacDonald MD MSc, Paul C. Hébert MD MHSc, Ken Flegel MDCM MSc, Matthew B. Stanbrook MD PhD

\section{References}

1. Olson EJ, Drage LA, Auger RR. Sleep deprivation, physician performance, and patient safety. Chest 2009;136:1389-96.

2. Committee on Optimizing Graduate Medical Trainee (Resident) Hours and Work Schedules to Improve Patient Safety, National Research Council. Ulmer C, Wolman DM, Johns MME, editors. Resident duty hours: enhancing sleep, supervision, and safety. Washington (DC): The National Academies Press; 2008

3. Volpp KG, Friedman W, Romano PS, et al. Residency training at a crossroads: dutyhour standards 2010. Ann Intern Med 2010;153:826-8.

4. Rothschild JM, Keohane CA, Rogers S, et al. Risks of complications by attending physicians after performing nighttime procedures. JAMA 2009;302:1565-72.

5. Malmberg B, Kecklund G, Karlson B, et al. Sleep and recovery in physicians on night call: a longitudinal field study. BMC Health Serv Res 2010;10:239.

6. Williamson AM, Feyer AM. Moderate sleep deprivation produces impairments in cognitive and motor performance equivalent to legally prescribed levels of alcohol intoxication. Occup Environ Med 2000;57:649-55.

7. Payette M, Chatterjee A, Weeks WB. Cost and workforce implications of subjecting all physicians to aviation industry work-hour restrictions. Am J Surg 2009;197:820-5.

Competing interests: See www.cmaj.ca/site/misc/cmaj_staff.xhtml.

Affiliations: See www.cmaj.ca/site/misc/cmaj_staff.xhtml.

Correspondence to: CMAJ editor, pubs@cmaj.ca

\section{CMAJ 2011. DOI:10.1503/cmaj.110402}

